\title{
Association between dental pulp stones and calcifying nanoparticles
}

\author{
This article was published in the following Dove Press journal: \\ International Journal of Nanomedicine \\ 7 January 2011 \\ Number of times this article has been viewed
}

\author{
Jinfeng Zengl* \\ Fang Yang ${ }^{*}$ \\ Wei Zhang ${ }^{2}$ \\ Qimei Gong' \\ Yu Du' \\ Junqi Ling \\ 'Department of Operative Dentistry \\ and Endodontics, Guanghua School \\ and Hospital of Stomatology and \\ Institute of Stomatological Research, \\ Sun Yat-sen University, Guangzhou, \\ China; ${ }^{2}$ National Neuroscience \\ Institute, Singapore \\ *These authors contributed equally to \\ this work
}

Correspondence: Junqi Ling Department of Operative Dentistry and Endodontics, Guanghua School and Hospital of Stomatology and Institute of Stomatological Research, Sun Yat-sen University, Guangzhou, China

Tel +86208386265l

Email lingjq@mail.sysu.edu.cn

\begin{abstract}
The etiology of dental pulp stones, one type of extraskeletal calcification disease, remains elusive to date. Calcifying nanoparticles (CNPs), formerly referred to as nanobacteria, were reported to be one etiological factor in a number of extraskeletal calcification diseases. We hypothesized that CNPs are involved in the calcification of the dental pulp tissue, and therefore investigated the link between CNPs and dental pulp stones. Sixty-five freshly collected dental pulp stones, each from a different patient, were analyzed. Thirteen of the pulp stones were examined for the existence of CNPs in situ by immunohistochemical staining (IHS), indirect immunofluorescence staining (IIFS), and transmission electron microscope (TEM). The remaining 52 pulp stones were used for isolation and cultivation of CNPs; the cultured CNPs were identified and confirmed via their shape and growth characteristics. Among the dental pulp stones examined in situ, $84.6 \%$ of the tissue samples staines positive for CNPs antigen by IHS; the corresponding rate by IIFS was $92.3 \%$. In $88.2 \%$ of the cultured samples, CNPs were isolated and cultivated successfully. The CNPs were visible under TEM as 200-400 nm diameter spherical particles surrounded by a compact crust. CNPs could be detected and isolated from a high percentage of dental pulp stones, suggesting that CNPs might play an important role in the calcification of dental pulp.
\end{abstract}

Keywords: calcifying nanoparticles, nanobacteria, pulp stones

\section{Introduction}

Frequently found on bitewing and periapical radiographs, pulp stones are discrete calcified bodies that are either located in the pulp tissue or attached to or embedded into dentine. Pulp stones are an oral disease with high incidence. It was reported that in young Australian adults, the prevalence of pulp stones was 46.1\% among dental patients who go to clinics and $10.1 \%$ of the teeth were affected. Occurrences were rare in premolars $(0.4 \%)$, but significantly higher in molars $(19.7 \%) .{ }^{1}$ The first and second molars were the teeth most commonly affected. ${ }^{2}$

Pulp stones may occur in one or all of the teeth in one person, even in unerupted or impacted teeth. Located in the pulp cavity or root canal, these stones often narrow or even obstruct the access to the apical point root canal. The calcification of pulp tissue might lead to the failure of root canal therapy and loss of the teeth.

The etiology of pulp stones has been controversial. Etiological factors implicated include aging, ${ }^{3}$ biological factors, ${ }^{4}$ physical factors,${ }^{5,6}$ and chemical factors. ${ }^{7,8}$

The discovery of calcifying nanoparticles (CNPs), also referred to as nanobacteria, led to novel theories for pathological calcification. CNPs first appeared as self-propagating calcifying macromolecular complexes found in bovine and human 
blood and blood products. These nanoparticles could produce biogenic carbonate apatite on their cell envelope at all growth phases, which resulted in white biofilm and mineral aggregates closely resembling those found in tissue calcification in the human body. ${ }^{9}$ CNPs are capable of producing nucleate hydroxyapatite; thus, they have been heralded as one potential etiological factor of pathological calcification, such as kidney stones or kidney cyst, ${ }^{10-18}$ gall stones, ${ }^{18-20}$ atherosclerosis, ${ }^{21-23}$ and dental calculus in periodontitis. ${ }^{24}$ As the lacking of satisfactory $16 \mathrm{~S}$ rRNA sequence, the controversy exists on the bacteria state on the so-called 'nanobacteria', so CNPs best describes the agent. ${ }^{25}$

Pulp stones are the characteristic manifestation of physiological or pathological calcification of the human body reflected in the dental pulp tissue. It could be hypothesized that CNPs are involved in the calcification of the dental pulp tissue.

\section{Materials and methods Collection of pulp stones}

Sixty-five randomly collected pulp stones, one from each patient, were scaled out by endodontists during root canal therapy (38 from maxillary molars and 27 from mandibular molars) in the Hospital of Stomatology, Sun Yat-sen University, Guangzhou, China. Ages of the patients ranged from 28 to 60 years. Sample information is listed in Supplementary Table 1. Written informed consent was obtained from each participant prior to enrollment, with approval of the ethical committee of the Hospital of Stomatology, Sun Yat-sen University. The pulp stones were collected over a period of 1 year. All of the dental pulp stones were kept in sterile normal saline before further treatments.

\section{Identification of pulp stones in situ}

Thirteen of the dental pulp stones were immersed in $4 \%$ paraformaldehyde and nitric acid for demineralization for 24-72 $\mathrm{h}$ and neutralized with 5\% sodium sulfate. After washing in water for $12 \mathrm{~h}$, each pulp stone, considered as one sample, was paraffin embedded and cut into 5 - $\mu \mathrm{m}$ sections. Three randomly selected tissue sections from each sample were prepared for immunohistochemical staining (IHS) and indirect immunofluorescence staining (IIFS). Each tissue section was deparaffinized, rehydrated, and demineralized and then processed as described earlier. ${ }^{9}$ IgG1 class anti-CNP mAbs, 8D10 (Nanobac Oy, Kuopio, Finland) was used as primary antibody in IHS and IIFS. The samples were examined under a Microphot-FXA microscope (Nikon Corporation, Tokyo, Japan) with fluorescence and differential interference contrast optics. Negative control sections underwent the same staining process, except that the primary mAb step was replaced with phosphate-buffered saline (PBS) buffer. Those with positive results were further examined with a transmission electron microscope (TEM; Hitachi H-600; Hitachi, Tokyo, Japan) operated at $15 \mathrm{kV}$, and micrographs were recorded.

\section{Culture of CNPs from pulp stones}

The remaining 52 pulp stones were randomly classified into 17 samples, with 3-4 for each sample. Samples were immersed in $0.3 \mu \mathrm{g} / \mathrm{mL}$ tetracycline and then demineralized in $1 \mathrm{~N} \mathrm{HCl}$ for $30 \mathrm{~min}$, then powdered and neutralized with 0.5 M Tris (pH 10.5; Sigma, St Louis, MO). Suspensions were centrifuged at 14,000 $\mathrm{g}$ for $15 \mathrm{~min}$ in a Minispin ${ }^{\circledR}$ Centrifuge and sterile filtered through $0.22-\mu \mathrm{m}$ millipore filters. The filtrate was cultured in flask containing Dulbecco's modified Eagle's medium (DMEM; GIBCO, Carlsbad, CA) with 10\% fetal bovine serum (FBS; Sigma) as described in previous studies. ${ }^{26}$ All FBS was $\gamma$-irradiated at a minimum dose of $30 \mathrm{kGy}$ at room temperature for $24 \mathrm{~h}$. DMEM incubated with FBS and Tris but without stone filtrate was used as negative controls. Subcultures were derived with a rubber scraper in serum-contained DMEM after 4 weeks of initial inoculation. All of the cultures were harvested by centrifugation at 20,000 $g$ for $45 \mathrm{~min}$ at $4^{\circ} \mathrm{C}$, washed with PBS (pH 7.2), and used for identification of CNPs.

\section{Culture examination}

The cultures were incubated for 8 weeks and evaluated microscopically for the growth of CNPs during and at the end of the incubation period. After incubation for 2, 4, or 8 weeks, the cultures were washed with PBS and photographed under inverted phase contrast microscope (Axiovert 40; Zeiss, Oberkochen, Germany). The cultures were examined by TEM to verify the detail on the morphology. For negative staining, samples were isolated by centrifugation at 20,000 $g$ for $1 \mathrm{~h}$ directly from FBS and diluted 1:5 in PBS. A carbon-coated 400-mesh copper grid was placed on a drop of CNP suspension in PBS for 1 min, washed with water, and stained on a drop of $1 \%$ phosphotungstic acid for $90 \mathrm{sec}$. Photographs were taken using TEM (JEM-100CXII; JEM, Tokyo, Japan) for morphological identification.

\section{Identification of CNPs from cultures}

All culture samples were harvested by $20,000 \mathrm{~g}$ centrifugation for $30 \mathrm{~min}$ at $4^{\circ} \mathrm{C}$ after 8 weeks of incubation. The pellets were analyzed for IHS and IIFS using CNP 
surface-antigen-specific mAb 8D10 to verify the existence of CNPs. In both IHS and IIFS, culture samples were spread on coverslips, fixed with $4 \%$ paraformaldehyde for $1 \mathrm{~h}$, incubated at $70^{\circ} \mathrm{C}$ for $10 \mathrm{~min}$, and blocked in $3 \% \mathrm{H}_{2} \mathrm{O}_{2}$ and $5 \%$ bovine serum albumin (BSA)-containing normal serum for $30 \mathrm{~min}$, respectively. In IHS, the particles were incubated with mouse monoclonal antinanobacteria antibodies 8D10 (NanoBac Oy) at $4{ }^{\circ} \mathrm{C}$ overnight; subsequently, the particles were sequentially incubated with biotinylated goat antimouse IgG antibody and streptavidin-biotin-peroxidase complex at $37^{\circ} \mathrm{C}$ for $30 \mathrm{~min}$ and visualized in diaminobenzidine solution for $10 \mathrm{~min}$. In IIFS, the cells were incubated with tetramethylrhodamine isothiocyanate (TRITC)-conjugated goat antimouse $\mathrm{IgG}$ antibody at $37^{\circ} \mathrm{C}$ for $30 \mathrm{~min}$; slides were then washed, dehydrated, and mounted for observation under a fluorescent microscope. In IHS and IIHS, the primary antibody was omitted in negative controls. Furthermore, von Kossa staining was applied to confirm the presence of mineralization. For von Kossa staining, cultures were harvested and reseeded into wells of a 24-well plate containing medium; after 24-h incubation, von Kossa staining was performed as described previously. ${ }^{27}$ Energy-dispersive X-ray analysis (EDX) were performed to show the topographic features by a scanning electron microscope (Quanta400; Philip FEI, Amsterdam, The Netherlands) equipped with EDX (INCA, Oxford, UK) as previously described. ${ }^{9}$

\section{Results}

\section{Identification of CNPs in pulp stones in situ}

Eleven out of the 13 tissue samples (84.6\%) stained positive for CNPs antigen for IHS, whereas by 12 positive samples were detected by IIFS (92.3\%). Positive staining results for IHS, as a brown-colored precipitate at the antigen site, are shown in Figure 1A. To negative result is shown in Figure 1a. The red-fluorescence-labeled signals of positive results for IIFS are shown in Figure 1B, and the corresponding negative result is shown in Figure 1b. TEM identified a large number of spherical coccoid particles, from 200 to $400 \mathrm{~nm}$, as shown in Figure $1 \mathrm{C}$, and the negative control is shown in Figure 1c.

\section{Morphology of CNPs in culture}

CNPs were successfully cultured from 15 of the 17 samples (see 'Materials and methods') after incubation for 2 weeks, demonstrating a positive rate of $88.2 \%$. The CNPs were first seen as individually separated and shaped typically as coccoid, bacilliform, or dumbbell with a highly refractile crust after 2 weeks, culture (Figure 2A). Those particles were very tiny with a diameter of 0.125 to $0.05 \mathrm{~mm}$ and barely detectable (Figure 2a). In 4 weeks, particles grew bigger and the average diameter was about $0.10 \mathrm{~mm}$ (Figure 2B, b). After 6-8 weeks, larger carbonate particles were aggregated in clumps and easily observed under the microscope. They were shown as coccoid particles with a diameter of $0.30-0.40 \mathrm{~mm}$ and aggregated in crowds (Figure 3A, B, and C). Some of them nearly fused together to form 'concentric circles' (Figure 3D, F) with a diameter of nearly $1.25 \mathrm{~mm}$. Surrounding the 'concentric circles', smaller size particles could be also seen (Figure 3E) and aggregated around relatively larger particles like 'satellites'.

\section{Culture examination}

By IHS, the harvested cultured particles exhibited a clearly visible yellow or brown staining with the anti-CNPs antibody 8D10 (Figure 4A), and no visible positive signal was detected in the negative controls (Figure 4a). Similarly, strong red fluorescent signals were detected by IIFS (Figure 4B). The same view under the light microscope is shown in Figure 4b. In the merged image under fluorescence and light microscope (Figure 4B +b), the thin arrow represents positive signals, and those represented by the thick arrow show the negative control. After von Kossa staining, the particles were colored black or brown, and their surrounding calcifying crust could be distinguished (Figure 4C). TEM image of the CNPs at 20,000× magnification, showing as 200-400 $\mathrm{nm}$ spherical particles (Figure 4D), and the topographic features on chemical composition of CNPs, were identified with calcium and phosphate peaks by EDX (Figure 4E).

\section{Discussion}

CNPs have spurred a major controversy in modern microbiology. Ambiguity of bacteriologic evidence for CNPs has led to various hypotheses of the nature and origin of CNPs, such as those of primordial life forms ${ }^{28-30}$ and those of inert mineral fetuin complexes. ${ }^{16,31,32}$ However, evidence for a significant role of CNPs in human biology has been accumulating.

CNPs were first identified in human kidney stones. ${ }^{33}$ Subsequently, a rat model of CNPs-induced kidney stones was successfully established. ${ }^{11}$ Since then, the significance of CNPs had extended substantially beyond kidney stones. Now, they are believed to be implicated in a variety of pathological calcifications in the human body.

Ciftcioglu et $\mathrm{al}^{34}$ suggested observation of mineralized fibers and numerous small lobular on the teeth with pulp stones, and if being cultured with nanobacterias (CNPs), 

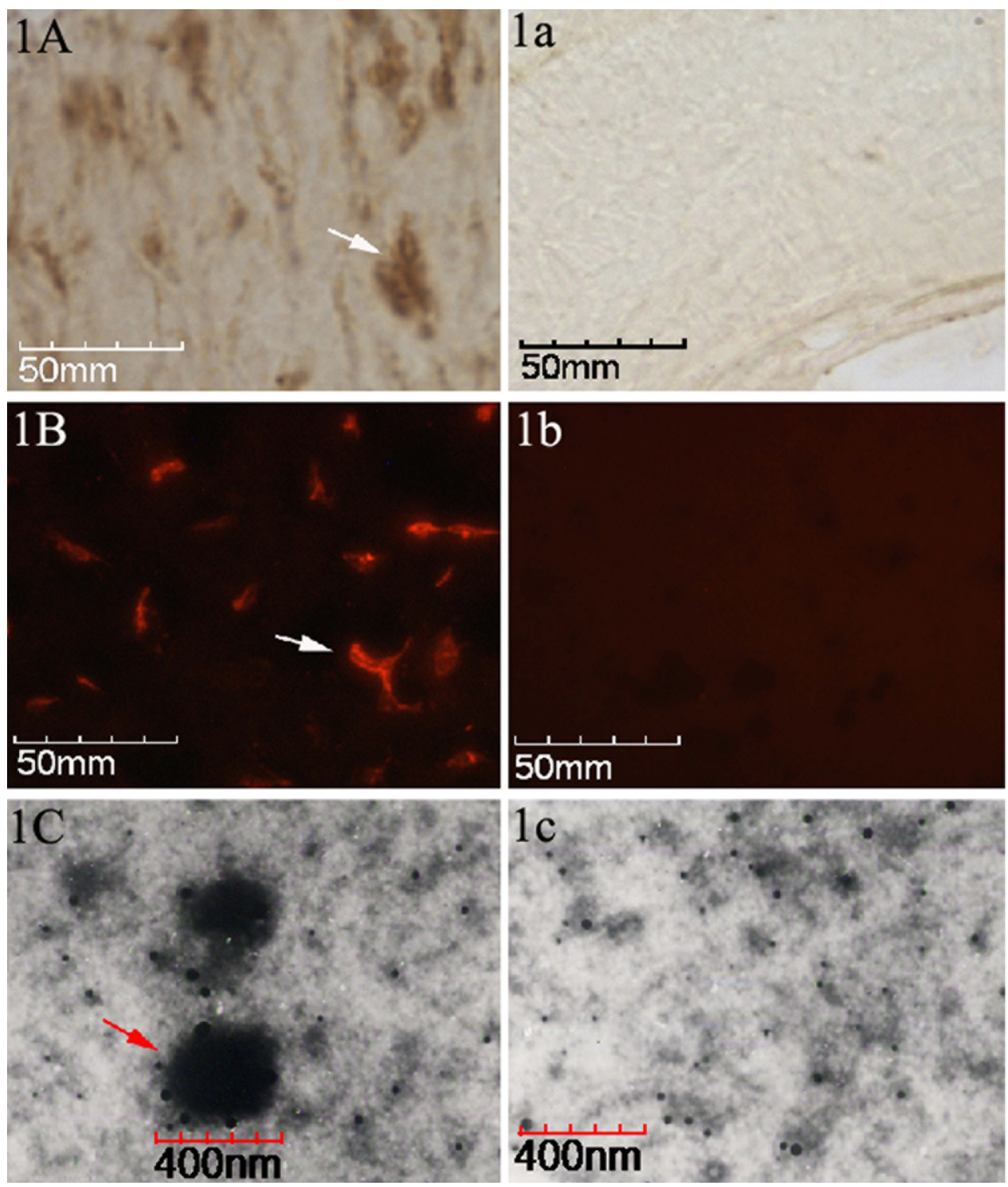

Figure I Identification of CNPs in paraffin-embedded pulp stone sections. A) The brown colored areas indicated by black arrows, by point indicate positive signals (existence of CNPs antigen) in the tissues by IHS. a) Negative control by IHS. B) Positive signals can be identified as red fluorescence by, IIFS. b) Negative control by IIFS. C) A TEM image at 35,000× zoom-in level shows the CNPs as spherical particles surrounded by a compact crust, 200-400 nm in diameter. c) Negative control in TEM image.

revealed voluminous mineral formation, resembling dental pulp stones. Based on Ciftcioglu's morphological observation, it could be hypothesized that CNPs might be implicated in the calcification of dental pulp stones. In the present study, in order to test this hypothesis, first, CNPs were identified in the dental pulp stones in situ. Furthermore, CNPs were isolated and characterized from the stone samples derived from cultures. Among the dental pulp stones examined in situ, the CNP-positive rates by IHS and IIFS analysis were $84.6 \%$ and $92.3 \%$, respectively. In $88.2 \%$ of the cultured samples,
CNPs were isolated and cultivated successfully. Therefore, the results suggested a potential link between the CNPs and human dental pulp stones.

Efforts were made in the present study to rule out of possibility of false positives of CNPs cultured from pulp stones. First, CNPs were reportedly found in $80 \%$ of the commercial FBS products. ${ }^{9}$ In this study, high dose of $\gamma$-ray $(30 \mathrm{kGy})$ radiation was applied for FBS to destroy the FBS-contained CNPs and thus to avoid false positive results. ${ }^{35,36}$ Second, FBS contained inhibitors on apatite 


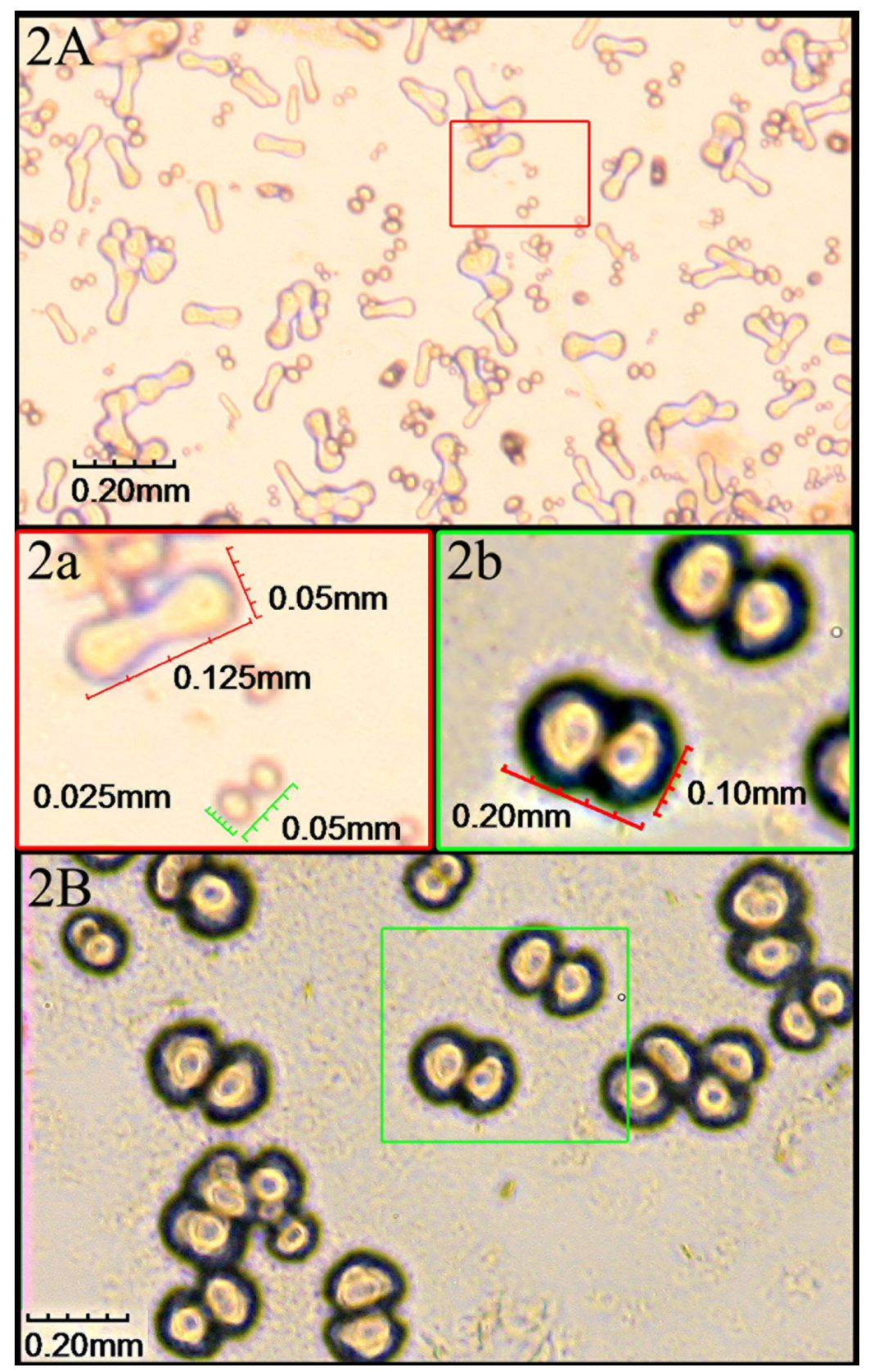

Figure 2 Cultures of CNPs observed through inverted phase contrast microscopy at 2-4 weeks. A) Particles were individually separated and were typically in coccoid, bacilliform, or dumbbell shape with a highly refractile crust after 2-week culture. a) Enlarged picture of (A) showing the particles were very tiny with a diameter of 0.025-0.05 mm. B) Particles grew bigger after 4-week culture. b) Enlarged picture of (B) showing the average diameter was about $0.10 \mathrm{~mm}$.

crystal formation, such as osteopontin, osteocalcin, and fetuin. $^{35,37}$ These inhibitors might interfere with the mineralization and, therefore, the visualization of CNPs. In this study, heat-inactivated FBS was used to remove the inhibitors of apatite crystal formation and avoid false negative results. Third, it was of great importance to ensure the CNPs isolated in this study were from pulp stone samples, not from other contaminating sources. Three anticontamination measures were taken. 1) All dental pulp stones used for the isolation of the CNPs were initially immersed in tetracycline for 30 min. 2) All reagents and instruments were sterilized, and rubber dam were utilized in clinical treatment. 3) The supernatants of CNPs cultures were filtered through a $0.22-\mu \mathrm{m}$ minipore filter, a generally accepted method to remove the common bacteria, fungi, and mycoplasma. Fourth, no other cellular entities were present as proved by microscopy and culture tests, and no cloudiness of color or $\mathrm{pH}$ changes were observed in medium. Therefore, it could be concluded that CNPs were, for the first time, isolated and identified from dental pulp stones. 


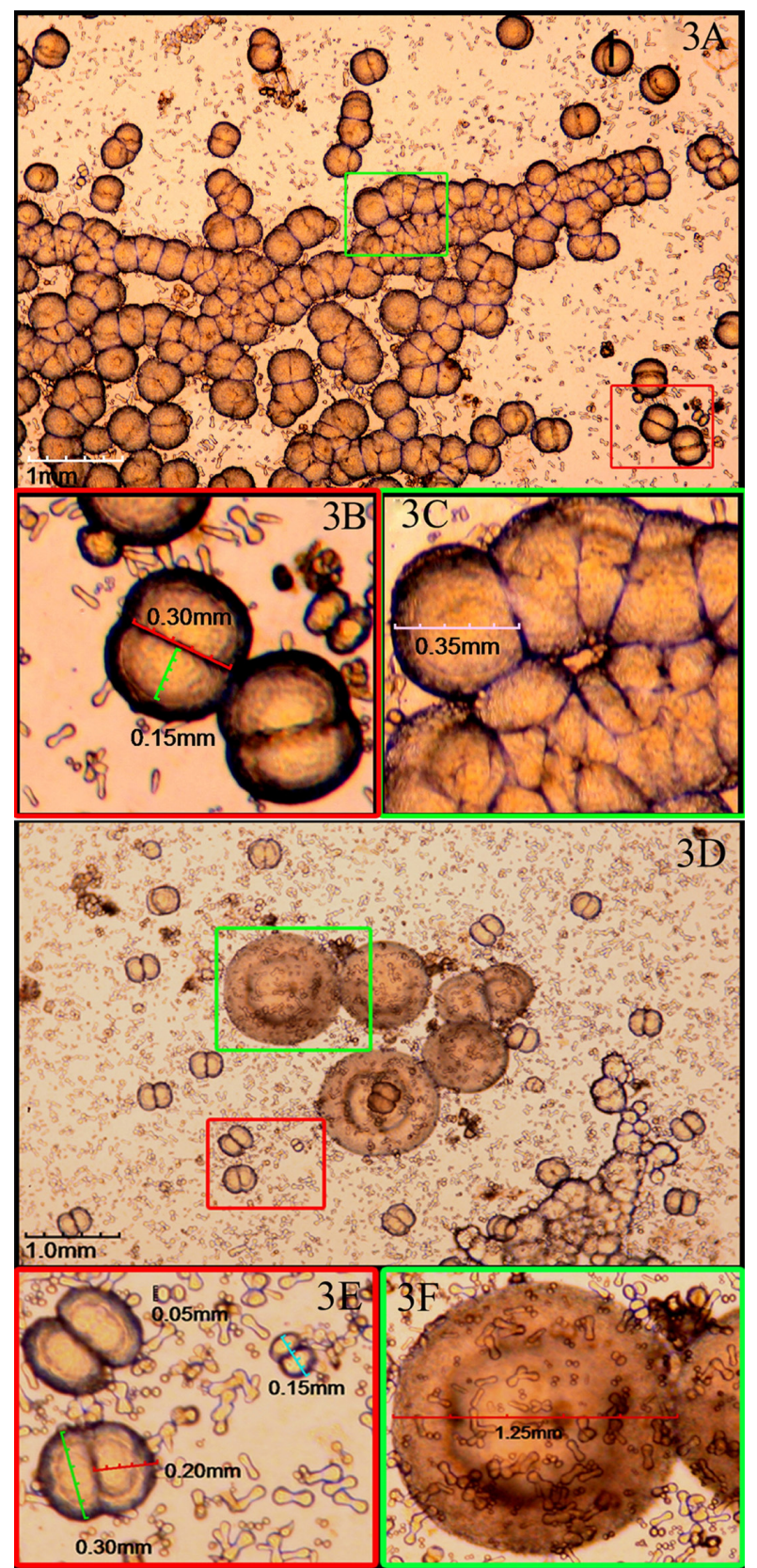

Figure 3 Viewing CNPs in particular culture stages at 6-8 weeks. A) Larger particles aggregated in clumps after 6-8 weeks of culture. B) and C) Enlarged pictures of (A) showing coccoid particles with a diameter of $0.30-0.40 \mathrm{~mm}$ and aggregated in crowds. D) 'Concentric circles' by the fused particles. E) and F) Enlarged pictures of (D) showing the 'concentric circles' with a diameter of nearly $1.25 \mathrm{~mm}$ and surrounded by smaller particles like 'satellites'.

Zhang et $\mathrm{al}^{24}$ described the detection of CNPs in gingival crevicular fluid and dental calculus as refractive aggregates in clumps after 6-week culture and the formation of a white-colored layer visible to the naked eye after 2 months. Our study provided more detailed information on the culture of CNPs in different growing states. CNPs isolated in the present study were coccoid with a highly refractive shell, similar in size and morphology to the CNPs reported
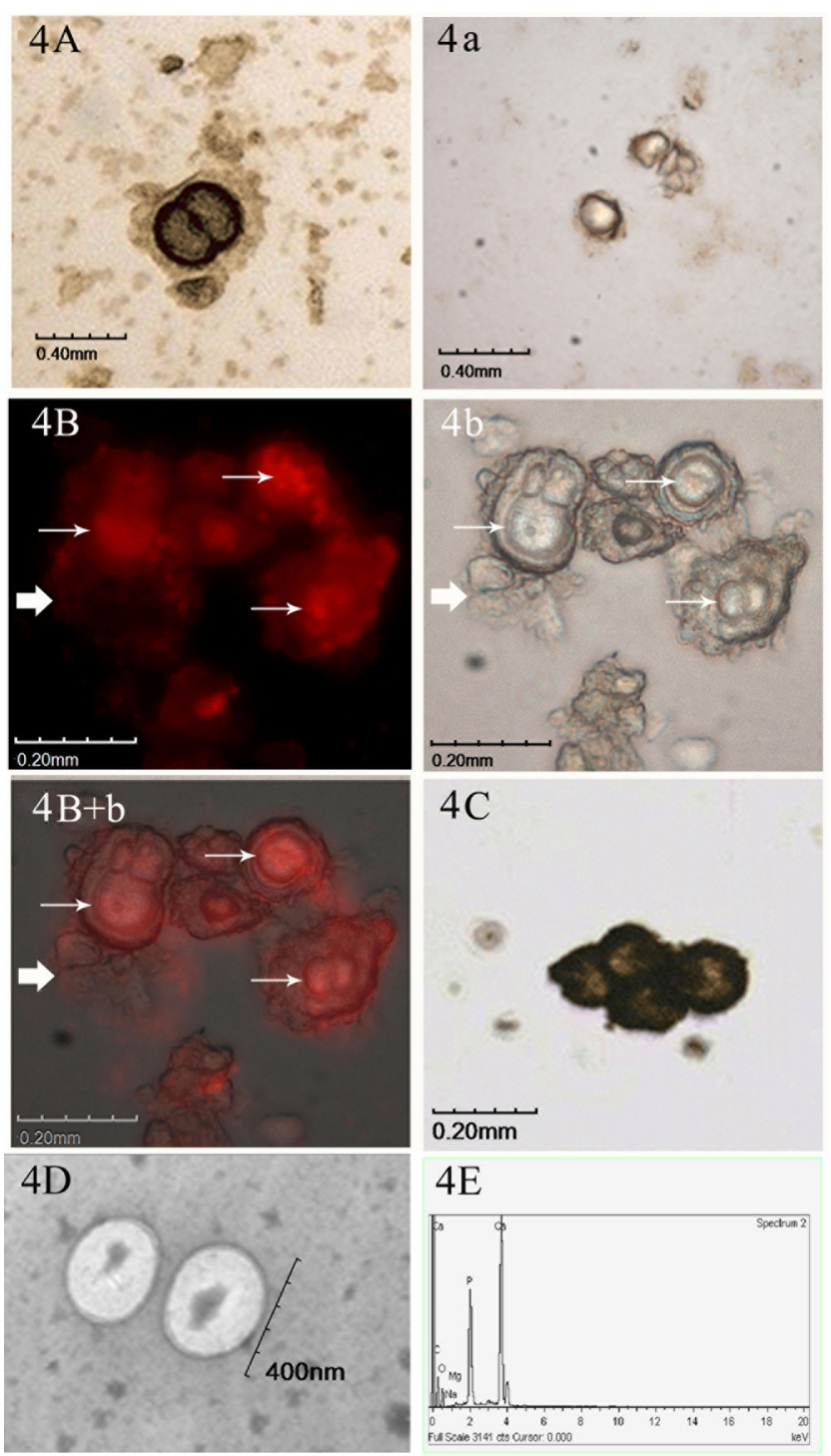

$4 \mathrm{E}$

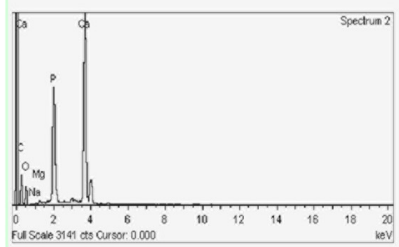

Figure 4 Identification of CNPs in cultures. A) IHS positive result using antiCNPs monoclonal antibody. a) Negative control by IHS. B) IIFS positive results of cultured CNPs using anti-CNPs monoclonal antibody. b) The same view under the light microscope by IIFS. B + b) Merged picture of image under fluorescence and light microscope. Thin arrow represents positive signals, and those represented by thick arrows show the negative control by IIFS. C) von Kossa staining of the CNPs. D) TEM image of the CNPs at 20,000× magnification, showing 200-400 nm spherical particles. E) Chemical analysis of CNPs by EDX showed calcium and phosphate peaks.

in other pathological calcification tissues in the human body. ${ }^{22,38,39}$ The morphological changes could be observed during the culture period. From the barely detectable tiny coccoid or dumbbell particles with a diameter of $0.025-0.05 \mathrm{~mm}$ after 2-week culture, these particles grew gradually to an average diameter of about $0.10 \mathrm{~mm}$ after 1 month. Furthermore, clumps aggregated in crowds were generated with average diameter of $0.30-0.40 \mathrm{~mm}$ in 6-8 weeks, and particles of all sizes could be observed. These observations indicated that CNPs were in a growing 
state and could bud off new particles unceasingly. In addition, two interesting phenomena in the cultures of CNPs from pulp stone were indicated.

First, 'concentric circles' formed after about 6-8 weeks of culture; this morphology has not been reported before for CNPs. It could be speculated that the individually separated CNPs not only aggregated into clusters, but also merged and exhibited this specific appearance. Coincidentally, one of the characteristic features of microcosmic structures observed in the dental pulp stone sections under scanning electron microscopy was also concentric circles. ${ }^{40,41}$ Thus, it is possible that the miniature form of concentric circles could be the very onset of dental pulp stone formation, and the gradual accumulation of calcium and phosphate around CNPs might lead to larger mineralization such as pulp stones.

Second, minute particles like 'satellites' aggregated around relatively larger particles, which could be observed in the subculture flasks. Interestingly, all these particles always appeared $\sim 24 \mathrm{~h}$ later in subcultures than those in primary cultures. It could be hypothesized that the subculturing procedure of filtering through $0.22-\mu \mathrm{m}$ minipore prevented the passage of larger particles or stripped them off the outer apatite crusts. As a result, CNPs in the subcultures were smaller and needed time to accumulate calcium and phosphate around their outer walls to become visible again.

In conclusion, this study has revealed the prevalence of CNPs in human dental pulp stones. The findings raised the possibility that CNPs might be implicated in the formation or development of dental pulp stones. Further studies are required to test whether CNPs are the causative agents of pulp stones or are simply a marker of the pathologic state. These investigations might lead to novel approaches for preventing or treating pulp stones and consequentially facilitating successful root canal therapy.

\section{Acknowledgment}

This work was supported by grants from the National Natural Science Foundation of China (No. 30471884).

\section{Disclosure}

The authors report no conflicts of interest in this work.

\section{References}

1. Ranjitkar S, Taylor JA, Townsend GC. A radiographic assessment of the prevalence of pulp stones in Australians. Aust Dent J. 2002;47(1): 36-40.

2. al-Hadi Hamasha A, Darwazeh A. Prevalence of pulp stones in Jordanian adults. Oral Surg Oral Med Oral Pathol Oral Radiol Endod. 1998; 86(6):730-732.
3. Hillmann G, Geurtsen W. Light-microscopical investigation of the distribution of extracellular matrix molecules and calcifications in human dental pulps of various ages. Cell Tissue Res. 1997;289(1): 145-154.

4. Yamazoe T, Aoki K, Simokawa H, Ohya K, Takagi Y. Gene expression of bone matrix proteins in a calcified tissue appeared in subcutaneously transplanted rat dental pulp. J Med Dent Sci. 2002;49(1):57-66.

5. Robertson A, Lundgren T, Andreasen JO, Dietz W, Hoyer I, Norén JG. Pulp calcifications in traumatized primary incisors. A morphological and inductive analysis study. Eur J Oral Sci. 1997;105(3):196-206.

6. Sübay RK, Kaya H, Tarim B, Sübay A, Cox CF. Response of human pulpal tissue to orthodontic extrusive applications. J Endod. 2001;27(8): 508-511.

7. Holtgrave EA, Hopfenmuller W, Ammar S. Tablet fluoridation influences the calcification of primary tooth pulp. J Orofac Orthop. 2001; 62(1):22-35.

8. Holtgrave EA, Hopfenmuller W, Ammar S. Abnormal pulp calcification in primary molars after fluoride supplementation. ASDC J Dent Child. 2002;69(2):126, 201-206.

9. Kajander EO, Ciftcioglu N. Nanobacteria: an alternative mechanism for pathogenic intra- and extracellular calcification and stone formation. Proc Natl Acad Sci U S A. 1998;95(14):8274-8279.

10. Jones JA, Ciftcioglu N, Schmid JF, Barr YR, Griffith D. Calcifying nanoparticles (nanobacteria): an additional potential factor for urolithiasis in space flight crews. Urology. 2009;73(1):210.e11-e13.

11. García Cuerpo E, Olavi Kajander E, Ciftçioglu N, et al. Nanobacteria. An experimental neo-lithogenesis model. Arch Esp Urol. 2000;53(4): 291-303.

12. Garcia Cuerpo E. Nanobacteria and urinary system lithiasis. Arch Esp Urol. 2003;56(2):191-192.

13. Jelic TM, Roque R, Yasar U, et al. Calcifying nanoparticles associated encrusted urinary bladder cystitis. Int J Nanomedicine. 2008;3(3): 385-390.

14. Kajander EO, Ciftcioglu N, Miller-Hjelle MA, Hjelle JT. Nanobacteria: controversial pathogens in nephrolithiasis and polycystic kidney disease. Curr Opin Nephrol Hypertens. 2001;10(3):445-452.

15. Lopez-Brea M, Selgas R. Nanobacteria as a cause of renal diseases and vascular calcifying pathology in renal patients ("endovascular lithiasis"). Enferm Infecc Microbiol Clin. 2000;18(10):491-492.

16. Drancourt M, Jacomo V, Lépidi H, et al. Attempted isolation of nanobacterium sp. microorganisms from upper urinary tract stones. J Clin Microbiol. 2003;41(1):368-372.

17. Shiekh FA, Khullar M, Singh SK. Lithogenesis: induction of renal calcifications by nanobacteria. Urol Res. 2006;34(1):53-57.

18. Li Y, Wen Y, Yang Z, et al. Culture and identification of nanobacteria in bile. Zhonghua Yi Xue Za Zhi. 2002;82(22):1557-1560.

19. Wen Y, Li YG, Yang ZL, et al. Detection of nanobacteria in serum, bile and gallbladder mucosa of patients with cholecystolithiasis. Chin Med J (Engl). 2005;118(5):421-424.

20. Wen Y, Li YG, Yang ZL, et al. Nanobacteria in serum, bile and gallbladder mucosa of cholecystolithiasis patients. Zhonghua Wai Ke Za Zhi. 2003;41(4):267-270.

21. Piper C, Dreier J, Kleesiek K, Körfer R, Horstkotte D. Nanobacteria to promote degenerative aortic valve stenosis? J Heart Valve Dis. 2006 15(4):499-501.

22. Puskás LG, Tiszlavicz L, Rázga Z, Torday LL, Krenács T, Papp JG. Detection of nanobacteria-like particles in human atherosclerotic plaques. Acta Biol Hung. 2005;56(3-4):233-245.

23. Bratos-Pérez MA, Sánchez PL, García de Cruz S, et al. Association between self-replicating calcifying nanoparticles and aortic stenosis: a possible link to valve calcification. Eur Heart J. 2008;29(3): 371-376.

24. Zhang SM, Tian F, Jiang XQ, et al. Evidence for calcifying nanoparticles in gingival crevicular fluid and dental calculus in periodontitis J Periodontol. 2009;80(9):1462-1470.

25. Kajander EO. Nanobacteria - propagating calcifying nanoparticles. Lett Appl Microbiol. 2006;42(6):549-552. 
26. Ciftçioglu N, Björklund M, Kuorikoski K, Bergström K, Kajander EO. Nanobacteria: an infectious cause for kidney stone formation. Kidney Int. 1999;56(5):1893-1898.

27. Ueno A, Kitase Y, Moriyama K, Inoue H. MC3T3-E1-conditioned medium-induced mineralization by clonal rat dental pulp cells. Matrix Biol. 2001;20(5-6):347-355.

28. Sommer AP, McKay DS, Ciftcioglu N, Oron U, Mester AR, Kajander EO. Living nanovesicles - chemical and physical survival strategies of primordial biosystems. J Proteome Res. 2003;2(4):441-443.

29. Sommer AP, Miyake N, Wickramasinghe NC, Narlikar JV, Al-Mufti S. Functions and possible provenance of primordial proteins. J Proteome Res. 2004;3(6):1296-1299.

30. Sommer AP, Pavlath AE. Sealing porous nanovesicles - solutions inspired by primordial biosystems. J Proteome Res. 2003;2(5):558-560.

31. Raoult D, Drancourt M, Azza S, et al. Nanobacteria are mineralo fetuin complexes. PLoS Pathog. 2008;4(2):e41.

32. Young JD, Martel J, Young L, Wu CY, Young A, Young D. Putative nanobacteria represent physiological remnants and culture by-products of normal calcium homeostasis. PLoS One. 2009;4(2):e4417.

33. Dorrell S. Nanobacteria linked to kidney disease. Mol Med Today. 1999;5(9):373.

34. Ciftcioglu N, Ciftcioglu V, Vali H, Turcott E, Kajander EO. Sedimentary rocks in our mouth: dental pulp stones made by nanobacteria. Proc SPIE Int Soc Opt Eng. 1998;3441(7):130-135.
35. Ciftcioglu N, Peltarri A, Kajander EO. Extraordinary growth phases of nanobacteria isolated from mammalian blood. Proc SPIE Soc Int Opt Eng. 1997;3111:429-435.

36. Kajander EO, Bjorklund M, Ciftcioglu N. Mineralization by nanobacteria. Proc SPIE Soc Int Opt Eng. 1998;3441:86-93.

37. Kajander EO, Kuronen I, Akerman KK, Pelttari A, Ciftcioglu N. Nanobacteria from blood: the smallest culturable autonomously replicating agent on Earth. Proc SPIE Int Soc Int Opt Eng. 1997;3111: $420-428$.

38. Khullar M, Sharma SK, Singh SK, et al. Morphological and immunological characteristics of nanobacteria from human renal stones of a north Indian population. Urol Res. 2004;32(3):190-195.

39. Altundag K, Altundag O, Akyurek S, Atik MA. Possible association between nanobacteria and malignant microcalcifications in breast cancer. Breast J. 2006;12(3):287.

40. Le May O, Kaqueler JC. Scanning electron microscopic study of pulp stones in human permanent teeth. Scanning Microsc. 1991;5(1): 257-267.

41. Le May O, Kaqueler JC. Electron probe micro-analysis of human dental pulp stones. Scanning Microsc. 1993;7(1):267-271. 


\section{Supplementary material}

Table SI Sample information on the selected teeth for pulp stones collection

\begin{tabular}{|c|c|c|c|c|}
\hline Sample ID & Age & Gender & Teeth & Teeth state \\
\hline I & 38 & Female & 16 & Chronic pulpitis \\
\hline 2 & 44 & Male & 16 & Chronic pulpitis \\
\hline 3 & 45 & Male & 36 & Apical periodontitis \\
\hline 4 & 28 & Male & 17 & Apical periodontitis \\
\hline 5 & 29 & Female & 26 & Apical periodontitis \\
\hline 6 & 60 & Female & 26 & Apical periodontitis \\
\hline 7 & 60 & Female & 28 & Apical periodontitis \\
\hline 8 & 45 & Female & 47 & Apical periodontitis \\
\hline 9 & 39 & Male & 46 & Chronic pulpitis \\
\hline 10 & 44 & Male & 17 & Necrotic pulp \\
\hline II & 44 & Male & 17 & Apical periodontitis \\
\hline 12 & 42 & Female & 17 & Necrotic pulp \\
\hline 13 & 37 & Male & 16 & Chronic pulpitis \\
\hline 14 & 32 & Female & 47 & Chronic pulpitis \\
\hline 15 & 45 & Male & 46 & Chronic pulpitis \\
\hline 16 & 56 & Female & 36 & Chronic pulpitis \\
\hline 17 & 43 & Female & 18 & Chronic pulpitis \\
\hline 18 & 29 & Male & 36 & Chronic pulpitis \\
\hline 19 & 28 & Male & 47 & Chronic pulpitis \\
\hline 20 & 30 & Female & 47 & Cracked tooth \& Acute pulpitis \\
\hline 21 & 40 & Female & 16 & Apical periodontitis \\
\hline 22 & 37 & Female & 16 & Cracked tooth \& Acute pulpitis \\
\hline 23 & 32 & Male & 17 & Cracked tooth \& Acute pulpitis \\
\hline 24 & 35 & Male & 17 & Apical periodontitis \\
\hline 25 & 41 & Male & 27 & Cracked tooth \& Acute pulpitis \\
\hline 26 & 50 & Male & 27 & Apical periodontitis \\
\hline 27 & 54 & Female & 27 & Apical periodontitis \\
\hline 28 & 52 & Female & 36 & Apical periodontitis \\
\hline 29 & 49 & Male & 37 & Apical periodontitis \\
\hline 30 & 51 & Female & 28 & Apical periodontitis \\
\hline 31 & 46 & Male & 27 & Cracked tooth \& Acute pulpitis \\
\hline 32 & 37 & Male & 27 & Chronic pulpitis \\
\hline 33 & 39 & Male & 27 & Chronic pulpitis \\
\hline 34 & 40 & Female & 38 & Chronic pulpitis \\
\hline 35 & 39 & Female & 46 & Chronic pulpitis \\
\hline 36 & 60 & Female & 46 & Chronic pulpitis \\
\hline 37 & 56 & Female & 27 & Necrotic pulp \\
\hline 38 & 50 & Female & 47 & Apical periodontitis \\
\hline 39 & 49 & Male & 47 & Apical periodontitis \\
\hline 40 & 47 & Male & 16 & Apical periodontitis \\
\hline 41 & 38 & Male & 27 & Chronic pulpitis \\
\hline 42 & 45 & Male & 37 & Chronic pulpitis \\
\hline 43 & 37 & Male & 47 & Apical periodontitis \\
\hline 44 & 38 & Male & 17 & Chronic pulpitis \\
\hline 45 & 43 & Female & 27 & Acute pulpitis \\
\hline 46 & 42 & Male & 47 & Apical periodontitis \\
\hline 47 & 39 & Female & 47 & Acute pulpitis \\
\hline 48 & 37 & Female & 46 & Acute pulpitis \\
\hline 49 & 36 & Female & 16 & Acute pulpitis \\
\hline 50 & 42 & Female & 17 & Necrotic pulp \\
\hline 51 & 38 & Female & 26 & Apical periodontitis \\
\hline 52 & 39 & Female & 26 & Apical periodontitis \\
\hline 53 & 59 & Male & 27 & Apical periodontitis \\
\hline
\end{tabular}


Table SI (Continued)

\begin{tabular}{lllll}
\hline Sample ID & Age & Gender & Teeth & Teeth state \\
\hline 54 & 50 & Male & 37 & Apical periodontitis \\
55 & 39 & Male & 36 & Apical periodontitis \\
56 & 54 & Male & 36 & Acute pulpitis \\
57 & 38 & Male & 27 & Acute pulpitis \\
58 & 38 & Female & 37 & Apical periodontitis \\
59 & 32 & Male & 28 & Acute pulpitis \\
60 & 45 & Female & 16 & Necrotic pulp \\
61 & 49 & Male & 37 & Apical periodontitis \\
62 & 48 & Male & Necrotic pulp \\
63 & 43 & Female & Necrotic pulp \\
64 & 44 & 17 & Apical periodontitis \\
65 & 52 & Male & 17 & Apical periodontitis \\
\hline
\end{tabular}

\section{Publish your work in this journal}

The International Journal of Nanomedicine is an international, peerreviewed journal focusing on the application of nanotechnology in diagnostics, therapeutics, and drug delivery systems throughout the biomedical field. This journal is indexed on PubMed Central, MedLine, CAS, SciSearch $\AA$, Current Contents ${ }^{\circledR} /$ Clinical Medicine,
Journal Citation Reports/Science Edition, EMBase, Scopus and the Elsevier Bibliographic databases. The manuscript management system is completely online and includes a very quick and fair peer-review system, which is all easy to use. Visit http://www.dovepress.com/ testimonials.php to read real quotes from published authors. 